\title{
Axis and Voltage ECG Assessment
}

National Cancer Institute

\section{Source}

National Cancer Institute. Axis and Voltage ECG Assessment. NCI Thesaurus. Code C111132.

An electrocardiographic assessment of the mean cardiac electrical vector and ECG voltage. 